\title{
Anticipatory postural adjustments in the shoulder girdle in the reach movement performed in standing by post-stroke subjects
}

\author{
Cláudia F. Silva ${ }^{a}$, Soraia Pereira ${ }^{b}$, Cláudia C. Silva ${ }^{b}$, Sílvia Ferreira ${ }^{a}$, Nuno Oliveira ${ }^{c}$ and Rubim Santos ${ }^{d}$

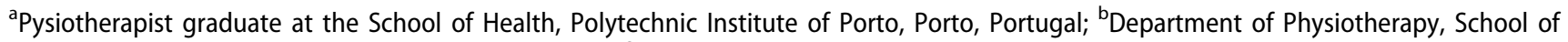 \\ Health, Polytechnic Institute of Porto, Porto, Portugal; 'Department of Physics, School of Health, Polytechnic Institute of Porto, Porto, \\ Portugal; ${ }^{d}$ Department of Physics and Activity and Human Movement Study Center (CEMAH), School of Health, Polytechnic Institute of \\ Porto, Porto, Portugal
}

\begin{abstract}
After a stroke in middle cerebral artery territory, there is a high probability of dysfunction of the ventromedial pathways, mainly related with postural control mechanisms such as the anticipatory postural adjustments (APAs). According to neuroanatomical knowledge, these pathways have a predominant ipsilesional disposition, which justifies a bilateral postural control dysfunction, often neglected in rehabilitation. In order to assess this bilateral postural control dysfunction, electromyography activity was assessed in eight post-stroke and 10 healthy individuals in the anterior deltoids, the superior and lower trapezius, and the latissimus dorsi as they reached for a bottle with both upper limbs separately at a self-selected velocity and fast velocity while standing associated with trunk kinematics analysis. Through this analysis it was possible to compare the timing of APAs in scapular muscles between sides in post-stroke and with healthy individuals, and to verify if there is a relation between the timing and the displacement of the trunk in the temporal window of the APAs. Indeed, post-stroke individuals show a delayed activation of APAs on scapular girdle muscles on both ipsilesional and contralesional sides, which were not reflected in the trunk displacement.
\end{abstract}

KEYWORDS Anticipatory postural adjustments; stroke; reach movement; standing; scapular stabilisers

\section{Introduction}

Reaching while standing is an essential task of daily life that requires spatial and temporal coordination to efficiently integrate the intended movement with appropriate postural control (McCombe Waller et al. 2016). This is a unique challenge to the central nervous system (CNS) that has to coordinate anticipatory postural adjustments (APAs) by anticipatory recruitment of the postural muscles, $100 \mathrm{~ms}$ to $50 \mathrm{~ms}$ prior to muscle activation.

Studies about APAs in reaching while standing have highlighted an initial inhibition of the soleus muscle, followed by the activation of the tibialis anterior (McCombe Waller et al. 2016). On the other hand, prior to the activation of the anterior deltoid (AD), the lateral gastrocnemius, the biceps femoral and the erector of the spine are simultaneously activated with the rotator cuff muscles (Dietz et al. 2000; Tyler and Karst 2004). Although previous studies have only focused on the lower limbs during reaching while standing, the task also demands a modification of the upper trunk orientation (Dickstein et al. 2004), including the scapula. With a better understand of the shoulder and the surrounding structures the scapula role in promoting optimal shoulder complex function with interaction of scapulohumeral anatomy and biomechanics in order to produce efficient movement has become recognized. To allow for the stability of shoulder movement, a coordinated activity of the surrounding musculature must be recruited in an anticipatory manner (Kibler et al. 2013) to allow the normal function of the glenohumeral joint (Paine and Voight 2013).

After a stroke the anticipatory postural activity (APA) may be compromised. Indeed, unilateral strokes in the territory of the middle cerebral artery (MCA) cause subcortical lesions, resulting in injury of the internal capsule and the corticofugal fibres that connect with the pontomedullary reticular formation, which is responsible for APAs (McCombe Waller et al. 2016). Injury in the corticoreticular connections prompts dysfunctions in the ventromedial systems, including the corticoreticular pathway, which could be associated with impairment of APAs on either the ipsilateral or contralateral side of the injured hemisphere (Matsuyama et al. 2004). In fact, considering the ventromedial system disposal, which, although bilateral, has a predominant ipsilateral projection, the ipsilesional deficits can no longer be ignored by the clinical and research community. That dynamic was identified by Pereira et al. (2014), who verified a delayed onset of APAs in the scapular stabilizers on both sides of the body among post-stroke individuals during sitting reach movement. However, standing is a more complex and challenging mechanical task, given the relatively high location of the centre of mass and the small area of support (Piscitelli et al. 2016). It becomes even more challenging after a stroke, due to larger postural sway and weight-bearing asymmetry 
(Sheikh, et al. 2017). Standing itself represents a perturbation to these individuals, but standing while reaching a bottle may lead to further disturbance (Tessem et al. 2007), leading to even more noticeable changes in timing of activation of APAs when compared to the seated position.

Understanding the behaviour of APAs among post-stroke individuals is crucial. The delayed onset in scapular stabilizers activation can interfere not only with stability, but also with the orientation of the upper limb (Van Der Fits and HaddersAlgra 1998; Tyler and Karst 2004; Jacobs et al. 2009). In fact, if the scapula fails to perform its stabilization role, shoulder function is compromised, which can result not only in a poor postural alignment of the trunk, but also may predispose to shoulder injury (Voight and Thomson 2000). This may be the basis for the development of post-stroke shoulder pain (Dabholkar et al. 2015). The shoulder girdle cannot be viewed as a separate segment, since it is attached to the spine via the trunk musculature and it is influenced by the neuromuscular activity of the trunk (Gjelsvik and Syre 2016). This dynamic process is especially important in neurorehabilitation, in which the recovery of upper limb function is paramount, once the use of the upper limb is vital to many activities of daily living, as well as to socialization and healthrelated quality-of-life (Harris et al. 2010).

Therefore, the aims of this study were to: (i) compare the timing of APAs in ipsilateral and contralateral scapular muscles during a common task of daily life (i.e., the reach movement while standing) in post-stroke and healthy individuals; (ii) compare the timing of APAs in ipsilesional and contralesional scapular muscles in post-stroke individuals; and (iii) verify if there is a relation between the activation timing of the stabilizers of the scapula and the displacement of the trunk in the temporal window corresponds to the APAs.

\section{Methods}

\section{Participants}

The study involved a control group of healthy individuals $(n=10)$ and a group of post-stroke individuals $(n=8)$. In the control group, the exclusion criteria included the presence of visual impairments, pain, and orthopaedic changes in the cervical spine or in the upper limb (Matias et al. 2006; Prange et al. 2010).

Inclusion criteria for the post-stroke group were vascular injury in the territory of the MCA up to 6 months after a stroke (Prange et al. 2010) and subsequent cortical or subcortical lesion (Dickstein et al. 2004), an ability to remain standing without assistance, an active range of motion of at least $15^{\circ}$ in the shoulder and elbow, and, finally, an ability to understand/follow oral instructions (Levin et al. 2002; Messier et al. 2006; Prange et al. 2010). In the post-stroke group, exclusion criteria were the presence of another neurological disorder (Kusoffsky et al. 2001), visual impairments, apraxia, shoulder pain lasting for 3 months or more (Prange et al. 2010), and cognitive impairment (scores less than 22 on the Mini Mental Test Examination) (Morgado et al. 2009).

\section{Instruments}

The Modified Barthel Index was used to assess post-stroke participants' independence in activities of daily life, whereas the Modified Tardieu Scale was used to assess their muscular resistance to passive movement. Surface electromyography (EMG) using the bioPLUX System ${ }^{\circledR}$ (Lisbon, Portugal) was applied to record muscular activity. AcqKnowledge 3.9.0 (BIOPAC Systems, Inc., Goleta, CA), with a sampling of $1000 \mathrm{~Hz}$, was used to analyse and process EMG data. Kinematic components were assessed using the Qualisys Track Manager System (Qualisys, Göteborg, Sweden), with a sampling frequency of $100 \mathrm{~Hz}$.

\section{Procedures}

A questionnaire was administered for characterization of the sample. Also, the individuals with post-stroke were assessed by the Modified Barthel Index and Tardieu scale.

For the task, participants remained standing with their upper limbs at rest, the shoulder joints stable in the neutral position, the elbows in extension, and forearm and wrist in the neutral position (Tyler and Karst 2004). The task 'reaching while standing' consisted of trying to reach a plastic bottle containing $0.5 \mathrm{~L}$ of water and return to the starting position without grasping (Bonnefoy et al. 2009; Kusoffsky et al. 2001; Shumway-Cook and Woollacott 2007). The bottle was placed in the scapular plane $30^{\circ}$ from the frontal plane of the individual at a midsternal height (Michaelsen et al. 2001) and a distance that corresponded to the participant's upper limb length (Kusoffsky et al. 2001; Michaelsen et al. 2001). The upper limb length was measured from the acromion to the metacarpophalangeal joint of the index finger (Reisman and Scholz 2006). Each participant performed three repetitions at a self-selected velocity (SSV) and a faster velocity (FV) after a constant verbal command; 'Reach the bottle' for SSV and 'Reach the bottle as fast as you can' for FV. Between repetitions, each participant rested for $2 \mathrm{~min}$ (Matias et al. 2006).

EMG activity was recorded on the $A D$, considered the agonist of the movement in the task (i.e., prime mover), the upper trapezius (UT), the lower trapezius (LT) (Matias et al. 2006; Yoshida et al. 2008; Bonnefoy et al. 2009), and the latissimus dorsi (LD). On LD, electrodes were placed obliquely $4 \mathrm{~cm}$ below the lower angle of the scapula (Escamilla et al. 2006; Herrington and Horsley 2009), i.e., on the muscular curvature at T12 level and along the line joining the posterior axillary to the spinous process of S2. For the other muscles, the references of the SENIAM project were used. Before the placement of the electrodes, the skin was prepared in order to maintain a skin-electrode impedance inferior to $5 \Omega$ (Correia and Mil-Homens 2004). Disposable electrodes with $10 \mathrm{~mm}$ diameter (Correia and Mil-Homens 2004; Matias et al. 2006) were placed bipolarly on the muscle belly parallel to the muscle fibres. The reference electrodes were placed bilaterally on the olecranon.

For synchronization of the kinematic and EMG signals, reflective markers were placed on the styloid apophysis of the radius and midsternal point (Levin et al. 2002; 
Michaelsen et al. 2004). Prior to data collection, the cameras were calibrated (Qualisys 2011). Movement velocity was calculated using the peak velocity of the marker on the styloid apophysis of the radius (Alt Murphy et al. 2006) by using Qualisys Track Manager software (Qualisys, Göteborg, Sweden).

\section{EMG analysis}

EMG data was analysed using AcqKnowledge. A 50- $\mathrm{Hz}$ highpass filter was used whenever visual inspection detected a cardiac muscle signal. The cardiac signal appeared similar in both groups, and, therefore, the same procedures were performed in each group. Also, a band-stop filter was used whenever the analysis of the raw signal spectrum indicated the presence of the signal $(50 \mathrm{~Hz})$ of the electric network. This analysis was done using Fast Fourier Transform of AcqKnowledge; both $50-\mathrm{Hz}$ high-pass filter and $50-\mathrm{Hz}$ bandstop filter were applied after the bandpass of $20-450 \mathrm{~Hz}$. The last step of processing was the application of root mean square (100 samples).

The latency for a specific muscle was defined as the instant lasting for at least $50 \mathrm{~ms}$ when its EMG amplitude was higher than the mean +2 SD of its baseline value, measured from -500 to $-450 \mathrm{~ms}$. The muscle latency was detected in a time window from -450 to $+200 \mathrm{~ms}$ in relation to T0 by a combination of computer algorithm and visual inspection of the averaged trials (Santos et al. 2010). Timing of muscle activation was described as the difference between the onset of activity of the muscles and the onset of AD activity (Dickstein et al. 2004; Wagner et al. 2007). The timing of activation reported in this study was the median value of the timing of activation in the three trials.

The displacement of the trunk defined by the marker placed on the midsternal point (Levin et al. 2002) was evaluated in the interval of $-100 \mathrm{~ms}$ to $-50 \mathrm{~ms}$ after the initiation of AD activity.

\section{Statistics}

Data analysis was performed with Predictive Analytics SoftWare (PASW) Statistics 18 (IBM SPSS, Chicago, IL) with $p$ values $<0.05$ considered statistically significant. Descriptive statistics were used to describe the characteristics of the sample.

To test the normality of the data of the control group, the Shapiro-Wilk test was used. Assuming the normality, a $t$-test was used to compare intra-group data. The Wilcoxon test was used for intra-group analysis in the post-stroke group. For inter-group analysis, the Mann-Whitney test was used. The correlation between the timing of activation and trunk displacement was established through the Spearman's correlation coefficient.

\section{Ethics}

Informed consent was obtained from all participants included in the study. All procedures performed in this study were in accordance with the ethical standards of the ethics committee of the School of Health of the Polytechnic Institute of Porto and with the 1964 Helsinki declaration.

\section{Results}

The control group (three males and seven females) had a median age of $51.5 \pm 5$ years, and the post-stroke group (six males and two females) had a median age of $60.5 \pm 5$ years (Table 1). All participants obtained a score of less than 1 point using the Tardieu scale.

With the sole purpose of characterization of the movement, the mean values of the maximal peak velocity were obtained. In the control group, the movement at SSV was performed with peak velocity of $1.62 \pm 0.299 \mathrm{~m} / \mathrm{s}$ and $1.58 \pm 0.254 \mathrm{~m} / \mathrm{s}$ in the dominant limb (DL) and non-dominant limb (NDL), respectively. On the other hand, the movement at FV was performed with peak velocity of $2.4 \pm 0.361$ $\mathrm{m} / \mathrm{s}$ in the DL and $2.4 \pm 0.337 \mathrm{~m} / \mathrm{s}$ in the NDL. Among poststroke participants, the movement at SSV was performed at $0.895 \pm 0.366 \mathrm{~m} / \mathrm{s}$ and $1.29 \pm 0.529 \mathrm{~m} / \mathrm{s}$ when performed by contralesional limb (CL) and ipsilesional limb (IL), respectively. Finally, the movement at FV had a peak velocity of $1.89 \pm 0.559 \mathrm{~m} / \mathrm{s}$ and $1.47 \pm 0.416 \mathrm{~m} / \mathrm{s}$ using the $\mathrm{CL}$ and $\mathrm{IL}$, respectively.

\section{Comparison of the onset of EMG activity on the ipsilateral and contralateral sides during the reaching movement performed by the control group}

No statistically significant difference was observed in the activation times of UT and LT by comparison between ipsilateral side and contralateral side to the movement performed by the DL at either SSV or FV (Table 2). However, statistically significant differences did emerge between the ipsilateral and contralateral LD in the DL in both SSV and FV. It was observed that the contralateral LD was activated before the

Table 1. Groups characterizations, considering the median (Mdn), interquartile deviation (IQR), and counts.

\begin{tabular}{|c|c|c|c|c|c|c|c|c|}
\hline Group & & Age (years) & G & Weight (Kg) & Height (m) & Stroke time (years) & Injured hemisphere & Barthel Index \\
\hline \multirow{4}{*}{ Healthy } & Md & 51.5 & - & 72.5 & 1.64 & - & - & - \\
\hline & IQR & 5 & - & 11.13 & 0.085 & - & - & - \\
\hline & Counts & - & $3 \mathrm{M}$ & - & - & - & - & - \\
\hline & & & $7 F$ & & & & & \\
\hline \multirow[t]{3}{*}{ Stroke } & Md & 60.5 & - & 81 & 1.695 & 2.5 & - & 89 \\
\hline & IQR & 5 & - & 12.63 & 0.065 & 1.19 & - & 6.25 \\
\hline & Counts & - & $\begin{array}{l}2 \mathrm{~F} \\
6 \mathrm{M}\end{array}$ & - & - & - & $\begin{array}{l}6 \text { Right } \\
2 \text { Left }\end{array}$ & - \\
\hline
\end{tabular}

G: Gender; F: female; M: male. 
Table 2. Comparison of the onset of EMG activity on the ipsilateral and contralateral sides during the reach movement performed at SSV and FV by the control group.

\begin{tabular}{|c|c|c|c|c|c|c|c|c|}
\hline & \multicolumn{4}{|c|}{ Dominant limb } & \multicolumn{4}{|c|}{ Non-dominant limb } \\
\hline & \multicolumn{2}{|r|}{ SSV } & \multicolumn{2}{|r|}{ FV } & \multicolumn{2}{|r|}{ SSV } & \multicolumn{2}{|r|}{$\mathrm{FV}$} \\
\hline & Mdn (IQR) & Test and $p$-value & Mdn (IQR) & Test and $p$-value & Mdn (IQR) & Test and $p$-value & Mdn (IQR) & Test and $p$-value \\
\hline UT Contra & $-58.5(54.5)$ & & $-48.5(25.8)$ & & $-34(35.4)$ & & $-51(31.6)$ & \\
\hline LT Ipsi & $-53(32.8)$ & $t=-0.982 ; p=0.176$ & $-32(14.4)$ & $t=0.007 ; p=0.498$ & $-65(39.4)$ & $t=-0.59 ; p=0.286$ & $-48.5(33.5)$ & $t=-1.384 ; p=0.1$ \\
\hline LT Contra & $-76(31.5)$ & & $-46(46.4)$ & & $-27(34.3)$ & & $-29.5(35.6)$ & \\
\hline $\begin{array}{l}\text { LD Ipsi } \\
\text { LD Contra }\end{array}$ & $\begin{aligned}-41.5 & (26) \\
70 & (24.9)\end{aligned}$ & $t=2.802 ; p=0.0105$ & $\begin{array}{r}-30.5(22.8) \\
-82(23.6)\end{array}$ & $t=1.846 ; p=0.049$ & $\begin{array}{l}-24.5(20.1) \\
-36.5(28.3)\end{array}$ & $t=0.618 ; p=0.276$ & $\begin{array}{c}38.5(33.5) \\
-54(31.1)\end{array}$ & $t=3.864 ; p=0.002$ \\
\hline
\end{tabular}

The mean (Md) and interquartile (IQR) deviations are in milliseconds. Positive values indicate that activation muscle after AD activation. Negatives values indicate that EMG activation occurred prior to AD.

Table 3. Comparison of the onset of EMG activity on the ipsilateral and contralateral sides during the reach movement performed by the contralesional and ipsilesional limbs at both velocities by the post-stroke group.

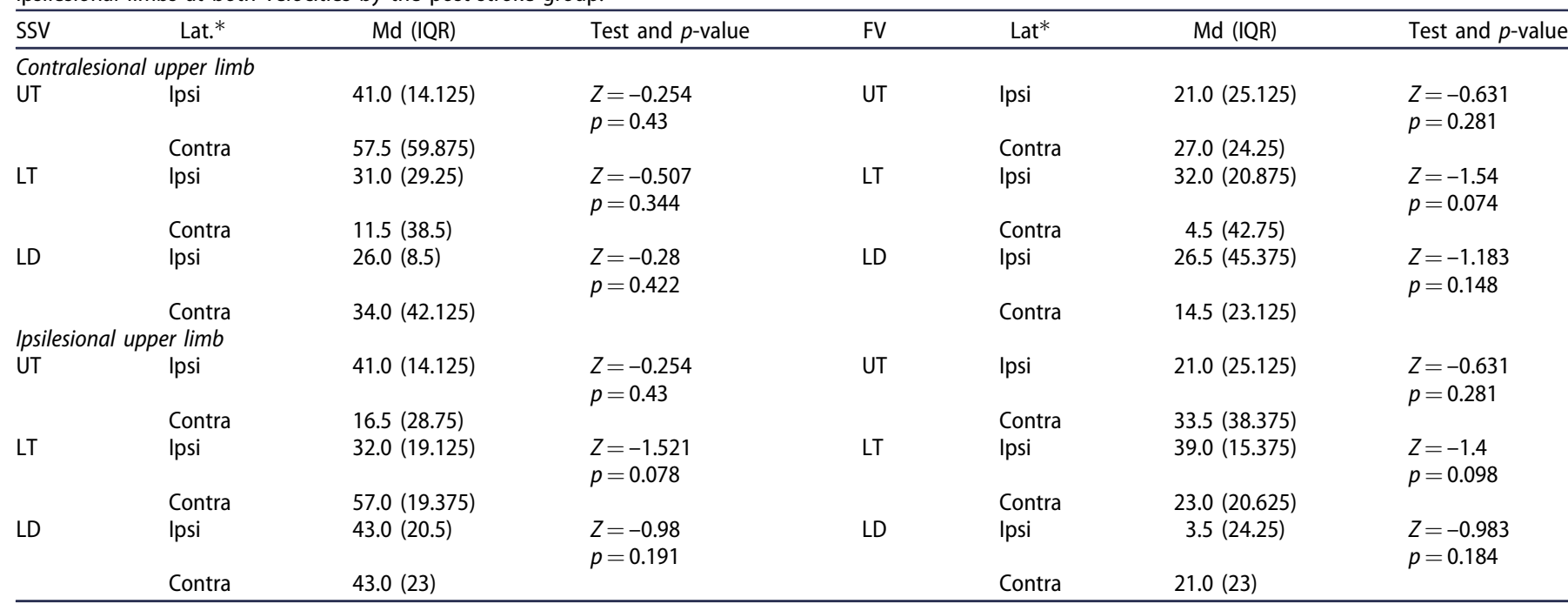

*Laterality to the movement.

The mean (Md) and interquartile (IQR) deviations are in milliseconds. Positive values indicate that activation of muscle is after $A D$ activation. Negative values indicate that EMG activation occurred prior to AD.

ipsilateral LD. The same behaviour was also observed when the movement was performed by the NDL at FV, but not at SSV.

Regarding the effect of movement velocity in both upper limbs, no statistically significant difference emerged between the timing of the activation of the muscles analysed. Differences between the peak movement velocity performed by the DL and NDL at SSV $(t=-0.394, p=0.351)$ and FV $(t=0.003, p=0.499)$ were also statistically not significant. Accordingly, for inter-group comparison, the activation values of the DL of the control group were used. The muscle activation onset times were compared only at SSV, because the velocity of movement used at SSV is more approximate to the velocity of movement used in daily life.

\section{Comparison of the onset of EMG activity on the ipsilateral and contralateral sides during the reaching movement performed by the post-stroke group}

No statistically significant difference emerged between the activation onset times of the ipsilateral and contralateral muscles to the movement performed by the $\mathrm{CL}$ at either SSV or FV (Table 3). Also, no statistically significant difference was verified when the movements were performed by the $\mathrm{IL}$ at either SSV or FV (Table 3).

Comparing the EMG activation times of the ipsilesional side with the contralesional side, no statistically significant difference was found, independently of the upper limb tested and the selected velocity.

Concerning the effect of velocity, no statistically significant difference was observed. Indeed, the muscle activation onset times during the movement performed by the $\mathrm{CL}$ at SSV when compared with the onset of the EMG activation of the same muscle in the movement performed at FV showed no statistically significant values (Figure 1).

Similarly, the muscle activation onset times during the movement performed by the IL did not change with the change of speed (Figure 2).

\section{Inter-group comparison of the timing of APAs while reaching at SSV}

The movement of the $\mathrm{CL}$ in the post-stroke group was compared to the movement of the $\mathrm{DL}$ in the control group. It verified a delay in the activation onset times the muscles analysed, namely contralateral UT $(U=10.0, p=0.006)$, 


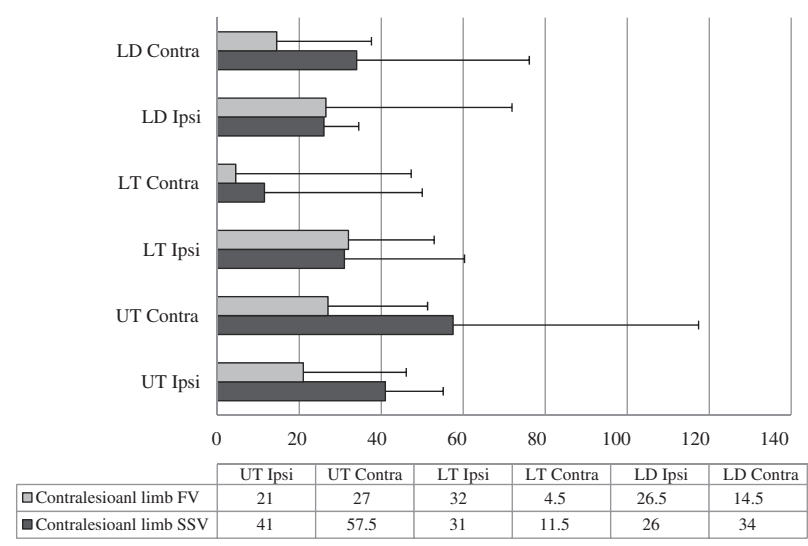

Figure 1. Comparison of the onset of electromyographic activation on the ipsilateral and contralateral sides during the reach movement performed at SSV and FV with contralesional limb by the post-stroke group.

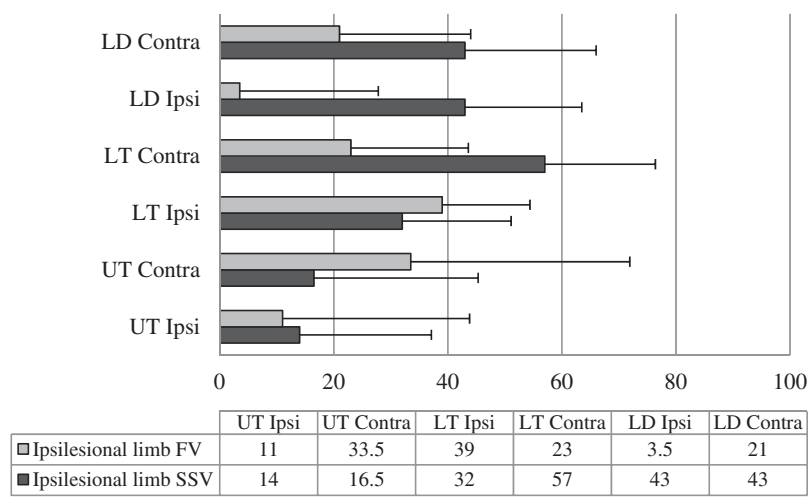

Figure 2. Comparison of the onset of electromyographic activation on the ipsilateral and contralateral sides during the reach movement performed at SSV and FV with ipsilesional limb by the post-stroke group.

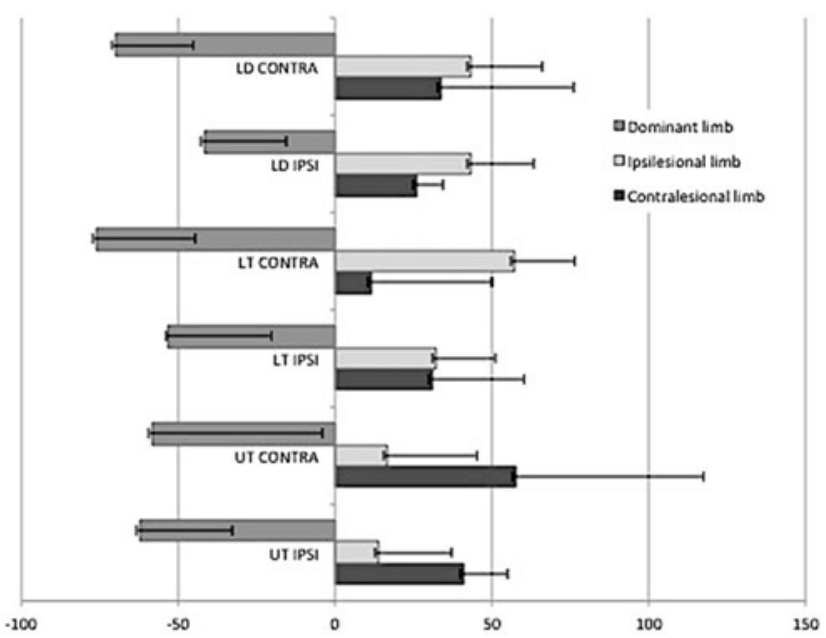

Figure 3. Inter-group comparison of the timing of anticipatory postural adjustments while reaching at the self-selected velocity by stroke group with the contralesional and ipsilesional limbs and by control group with the dominant limb. All relationships were statistically significative with a $p<0.05$. Contralateral (Contra) and ipsilateral (Ipsi) is in respect to movement performance.

ipsilateral UT $(\mathrm{U}=4.0, p<0.001)$ and $\mathrm{LT}(\mathrm{U}=3.0, p<0.001)$, contralateral LT $(U=7.0, p=0.001)$ and LD $(U=6.0$, $p=0.001)$, and ipsilateral $\mathrm{LD}(\mathrm{U}=6.0, p=0.001)$-as shown in Figure 3.
Delayed onset of muscle activation was also verified in the $\mathrm{IL}$ in the post-stroke group when compared with the $\mathrm{DL}$ in the control group-ipsilateral UT $(U=5.0, p<0.001)$ and LT $(\mathrm{U}=4.0, p<0.001)$, contralateral UT $(\mathrm{U}=10.0, p=0.003)$, contralateral $\mathrm{LT}(\mathrm{U}=7.0, p=0.001)$, ipsilateral $\operatorname{LD}(\mathrm{U}=8.0$, $p=0.002)$, and contralateral $\mathrm{LD}(\mathrm{U}=0, p<0.001)$-Figure 3 .

\section{Inter-group comparison of trunk displacement during reaching performed at SSV and its relationship with the timing of EMG activation}

Regarding trunk displacement, no statistically significant difference emerged in the distributions between the control and post-stroke groups. However, the median and interquartile deviation of the trunk displacement in the post-stroke group was $1.945 \pm 0.6 \mathrm{~mm}$ when the movement was performed by the $\mathrm{CL}(\mathrm{U}=26, p=0.287)$ and $1.4 \pm 0.61 \mathrm{~mm}$ in the movement performed by the IL $(\mathrm{U}=22, p=0.157)$.

The correlation analysis between the muscle activation onset times and trunk displacement in the post-stroke group, when the movement was performed by the $\mathrm{CL}$ showed no statistically significant correlation.

\section{Discussion}

The main finding in this study was the verification of a delay in the APAs activation bilaterally in the post-stroke individuals when compared to the healthy ones. In fact, all the muscles of post-stroke individuals tested in this study became active after the prime mover (i.e., $A D$ ), regardless of the upper limb used in the movement.

The delayed muscle activation was found in all muscles tested in both ipsilesional and contralesional sides when compared to healthy subjects. These results were in line with findings from a previous study carried out by Pereira et al. (2014), who also noted a delay in the latissimus dorsi and lower trapezius activation in both sides in post-stroke individuals. However, in Pereira et al.'s (2014) study, the task was performed in a seated position. In a sitting position, the base of support is substantially larger when compared to the standing position. Therefore, the task of maintaining the centre of mass projection within boundaries of the base of support, when seated, is less challenging as opposed to the task performed in a standing position. This explains why in the present study all the scapular muscles presented a delay in their activation.

Biomechanically, the task of reaching while standing is more complex and inherently unstable, due to factors such as the high location of the centre of mass, small support area, and multiple joints between the feet and the centre of mass. When a standing subject performs an upper limb movement and interacts with an external object, the mechanical coupling between body segments leads to postural perturbations that may compromise the stability. Some authors believe that APAs associated with a voluntary movement would be attenuated when the posture is unstable as a strategy of protection against the potential destabilizing effect induced by the centre of pressure shift during the 
APAs (Yiou et al. 2009). Although the task in study is relatively easy for the healthy subjects, the same does not apply to the post-stroke subjects for who the standing position is by itself a disturbance. This relies on the fact that these individuals have difficulty modulating the timing of the APAs.

The impairment of APAs in post-stroke individuals established in this study was also observed by Dickstein et al. (2004), who concluded that post-stroke individuals present a delayed onset in the anticipatory activity in the LD, external oblique, and rectus abdominal muscles on the contralesional side. Also, a delay was found in the onset activity of their participants' abdominal muscles on the ipsilesional side. Similarly, Slijper et al. (2002) concluded that APAs were reduced in post-stroke individuals on the contralesional side, with signs of decreased ability to modulate APAs in the homologous muscles on the ipsilesional side.

Different studies on APAs in post-stroke individuals seem to indicate impairment in APAs on the ipsilesional side. Most stroke lesions occur in the territory of the MCA, which indicates a high probability of damage of pathways with predominant ipsilesional disposition (Silva et al. 2017). The damage of the internal capsule compromises the output of the corticoreticular pathways, resulting in the consequent dysfunction of the ventromedial systems, namely the reticulospinal system (Mazevet et al. 2003; Luft et al. 2004). Although bilateral, the reticulospinal system has a predominant ipsilateral disposition that could explain the delayed activation of APAs on the ipsilesional side (Mazevet et al. 2003; Luft et al. 2004; Sousa et al. 2015).

The CNS can adapt the parameters of APAs to postural components of the task. Such adaptability makes movement efficient. In respect to the velocity of movement, studies have shown that anticipatory EMG activity lasts longer and has larger amplitude in the fast movements. In contrast, slow movements present a more variable sequence of anticipatory activity (Bouisset et al. 2000). According to Bouisset et al. (2000), a significant linear relationship occurs between the amplitude and the square of the velocity of movement. In the present study, a statistically significant difference was not verified in the change of speed between groups. Most studies have concluded a modulation of anticipatory EMG activity regarding amplitude, which supports the fact that the onset time of APAs could be insensitive to the modification of velocity.

Despite the observation of a delayed activation of APAs, no statistically significant difference was found in the trunk displacement in the temporal window of APAs between groups. Conversely, Pereira et al. (2014) proved a higher trunk displacement in the movement performed by the contralesional limb. Once again, these differences may be related with a single change of variable, the position of the subject. As standing is a source of perturbation, we hypothesize that post-stroke individuals recruit a larger trunk displacement, but not so excessive as in sitting, in order to maintain the projection of centre of mass in the support base and not to lose balance. Based on the descriptive statistics, it is possible to conclude that there is a larger displacement of the trunk when the movement is performed by $\mathrm{CL}$ as well as a verification of high variability among individuals. However, the absence of significant results can be related to the selected interval of time for analysis and the small sample of the study. In future studies, it would be important to understand the consequences of APA impairment on trunk orientation and stability assessed according to the displacement of the centre of pressure.

\section{Conclusion}

Post-stroke individuals with subcortical lesions in the territory of the MCA show a delayed pre-activation of scapular stabilizers on both ipsilesional and contralesional sides. The ability of post-stroke subjects to prepare for a self-initiated perturbation is impaired, resulting in a compromised adaptation for the ever-changing environmental requirements. Additionally, this study also highlights the importance of taking into account the ipsilesional side. This side was being considered for a long time to be unaffected by stroke injuries, and it had been used as a reference for comparison in order to identify dysfunctions on the contralesional side in clinical practice and research. From a clinical perspective, the results of the present study emphasize the importance of paying attention to the contralesional side as well as to the ipsilesional side in the rehabilitation of post-stroke individuals.

\section{References}

Alt Murphy M, Sunnerhagen KS, Johnels B, Willén C. 2006. Three dimensional kinematic motion analysis of a daily activity drinking from a glass: a pilot study. J Neuroeng Rehab. 3:18.

Bonnefoy A, Louis N, Gorce P. 2009. Muscle activation during a reach-tograsp movement in sitting position: influence of the distance. J Electromyogr Kinesiol. 19:269-275.

Bouisset S, Richardson J, Zattara M. 2000. Do anticipatory postural adjustments occurring in different segments of the postural chain follow the same organisational rule for different task movement velocities, independently of the inertial load value? Exp Brain Res. 132:79-86.

Correia PP, Mil-Homens P. 2004. A Electromiografia no Estudo do Movimento Humano (F. d. M. Humana. Ed.). Lisboa, Portugal.

Dabholkar A, Mehta D, Yardi S, Dabholkar T. 2015. Assessment of scapular behavior in stroke patients. Int J Health Rehabil Sci. 4:95-102.

Dickstein R, Shefi S, Marcovitz E, Villa Y. 2004. Anticipatory postural adjustment in selected trunk muscles in poststroke hemiparetic patients. Arch Phys Med Rehabil. 85:261-267.

Dietz V, Kowalewski R, Nakazawa K, Colombo G. 2000. Effects of changing stance conditions on anticipatory postural adjustment and reaction time to voluntary arm movement in humans. J Physiol (Lond). 524(Pt 2):617-627.

Escamilla RF, Babb E, DeWitt R, Jew P, Kelleher P, Burnham T, Busch J, D’Anna K, Mowbray R, Imamura RT. 2006. Electromyographic analysis of traditional and nontraditional abdominal exercises: implications for rehabilitation and training. Phys Ther. 86:656-671. 
Gjelsvik BB, Syre L. 2016. The bobath concept in adult neurology (2nd Edition). Stuttgart, Germany: Thieme.

Harris JE, Eng JJ, Miller WC, Dawson AS. 2010. The role of caregiver involvement in upper-limb treatment in individuals with subacute stroke. Phys Ther. 90:1302-1310.

Herrington L, Horsley I. 2009. Electromyographic analysis of selected shoulder muscles during a rugby football tackle. BMC Sports Sci Med Rehabil. 1:1-7.

Jacobs JV, Lou JS, Kraakevik JA, Horak FB. 2009. The supplementary motor area contributes to the timing of the anticipatory postural adjustment during step initiation in participants with and without Parkinson's disease. Neuroscience. 164:877-885.

Kibler WB, Ludewig PM, McClure PW, Michener LA, Bak K, Sciascia AD. 2013. Clinical implications of scapular dyskinesis in shoulder injury: the 2013 consensus statement from the 'Scapular Summit'. Br J Sports Med. 47:877-885.

Kusoffsky A, Apel I, Hirschfeld H. 2001. Reaching-lifting-placing task during standing after stroke: Coordination among ground forces, ankle muscle activity, and hand movement. Arch Phys Med Rehabil. 82:650-660.

Levin M, Michaelsen S, Cirstea C, Roby-Brami A. 2002. Use of the trunk for reaching targets placed within and beyond the reach in adult hemiparesis. Exp Brain Res. 143:171-180.

Luft AR, Waller S, Forrester L, Smith GV, Whitall J, Macko RF, Schulz JB, Hanley DF. 2004. Lesion location alters brain activation in chronically impaired stroke survivors. Neuroimage 21:924-935.

Matias R, Batata D, Morais D, Miguel J, Estiveira R. 2006. Estudo do Comportamento Motor dos Músculos Deltóide, Trapézio, e Grande Dentado Durante a Elevação do Braço em Sujeitos Asssintomáticos. EssFisioOnline 2:3-23.

Matsuyama K, Mori F, Nakajima K, Drew T, Aoki M, Mori S. 2004. Locomotor role of the corticoreticular-reticulospinal-spinal interneuronal system. Prog Brain Res 143:239-249.

Mazevet D, Meunier S, Pradat-Diehl P, Marchand-Pauvert V, PierrotDeseilligny E. 2003. Changes in propriospinally mediated excitation of upper limb motoneurons in stroke patients. Brain. 126:988-1000.

McCombe Waller S, Yang CL, Magder L, Yungher D, Gray V, Rogers MW. 2016. Impaired motor preparation and execution during standing reach in people with chronic stroke. Neurosci Lett. 630:38-44.

Messier S, Bourbonnais D, Desrosiers J, Roy Y. 2006. Kinematic analysis of upper limbs and trunk movement during bilateral movement after stroke. Arch Phys Med Rehabil. 87:1463-1470.

Michaelsen SM, Jacobs S, Roby-Brami A, Levin MF. 2004. Compensation for distal impairments of grasping in adults with hemiparesis. Exp Brain Res. 157:162-173.

Michaelsen SM, Luta A, Roby-Brami A, Levin MF. 2001. Effect of Trunk Restraint on the Recovery of Reaching Movements in Hemiparetic Patients. Stroke. 32:1875-1883.

Morgado J, Rocha CS, Maruta C, Guerreiro M, Martins IP. 2009. Novos Valores Normativos do Mini-Mental State Examination. SINAPSE: Publicações Da Sociedade Portuguesa De Neurologia 9:10-18.

Paine R, Voight ML. 2013. The role of the scapula. Int J Sports Phys Ther. 8:617-629.

Pereira S, Silva CC, Ferreira S, Silva C, Oliveira N, Santos R, Vilas-Boas JP, Correia MV. 2014. Anticipatory postural adjustments during sitting reach movement in post-stroke subjects. J Electromyogr Kinesiol. 24:165-171.

Piscitelli D, Falaki A, Cerri CG, Latash ML. 2016. Anticipatory synergy adjustments and anticipatory postural adjustments: Effects of predictability of perturbation direction. Gait Posture. 49:S3-S4.

Prange $G$, Jannink $M$, Stienen $A$, van der Kooij $H$, ljzerman $M$, Hermens H. 2010. An explorative, cross-sectional study into abnormal muscular coupling during reach in chronic stroke patients. J Neuroengineering Rehabil. 7:14.

Qualisys AB. 2011. QTM Qualisys Track Manager user manual. Gothenburg, Sweden: Qualisys AB.

Reisman D, Scholz J. 2006. Workspace location influences joint coordination during reaching in post-stroke hemiparesis. Exp Brain Res. 170:265-276.

Santos MJ, Kanekar N, Aruin AS. 2010. The role of anticipatory postural adjustments in compensatory control of posture: 1. Electromyographic analysis. J Electromyogr Kinesiol. 20:388-397.

Sheikh M, Azarpazhooh MR, Hosseini HA. 2017. The effect of immediate decreasing of weight bearing asymmetry on quiet standing postural control in individuals with chronic stroke. Physiother Theory Pract. 33:751-757.

Shumway-Cook A, Woollacott M. 2007. Motor Control: Translating Research into Clinical Practice. Third Edition. Philadelphia: Lippincott Williams \& Wilkins.

Silva A, Sousa ASP, Silva CC, Santos R, Tavares JMRS, Sousa F. 2017. The role of the ipsilesional side in the rehabilitation of post-stroke subjects. Somatosens Mot Res. 34:185-188.

Slijper H, Latash ML, Rao N, Aruin AS. 2002. Task-specific modulation of anticipatory postural adjustments in individuals with hemiparesis. Clin Neurophysiol. 113:642-655.

Sousa AS, Silva A, Santos R. 2015. Ankle anticipatory postural adjustments during gait initiation in healthy and post-stroke subjects. Clin Biomech (Bristol, Avon). 30:960-965.

Tessem S, Hagstrøm N, Fallang B. 2007. Weight distribution in standing and sitting positions, and weight transfer during reaching tasks, in seated stroke subjects and healthy subjects. Physiother Res Int. 12:82-94.

Tyler AE, Karst GM. 2004. Timing of muscle activity during reaching while standing: systematic changes with target distance. Gait Posture. 20:126-133.

Van Der Fits IB, Hadders-Algra M. 1998. The development of postural response patterns during reaching in healthy infants. Neurosci Biobehav Rev. 22:521-526.

Voight ML, Thomson BC. 2000. The role of the scapula in the rehabilitation of shoulder injuries. J Athl Train. 35:364-372.

Wagner JM, Dromerick AW, Sahrmann SA, Lang CE. 2007. Upper extremity muscle activation during recovery of reaching in subjects with post-stroke hemiparesis. Clinical Neurophysiology. 118:164-176.

Yiou E, Mezaour M, Le Bozec S. 2009. Anticipatory postural adjustments and focal performance during bilateral forward-reach task under different stance conditions. Motor Control. 13:142-160.

Yoshida S, Nakazawa K, Shimizu E, Shimoyama I. 2008. Anticipatory postural adjustments modify the movement-related potentials of upper extremity voluntary movement. Gait Posture. 27:97-102. 